\section{Prevalência de cárie radicular em adultos e idosos na região sudeste do Estado de São Paulo, Brasil}

\author{
Dental root surface caries prevalence \\ among adults and senior citizens in southeast \\ São Paulo State, Brazil
}

Lilian Berta Rihs 1

Maria da Luz Rosário de Sousa 1 Ronaldo Seichi Wada 1

\footnotetext{
1 Faculdade de Odontologia de Piracicaba, Universidade Estadual de Campinas, Piracicaba, Brasil.

Correspondência M. L. R. Sousa Departamento de Odontologia Social, Faculdade de Odontologia de Piracicaba, Universidade Estadual de Campinas. Av. Limeira 901, Piracicaba, SP 13414-018, Brasil. luzsousa@fop.unicamp.br
}

\begin{abstract}
In recent decades there has been an increase in the occurrence of dental root surface caries, especially among adults and senior citizens. This study aimed to identify root surface caries prevalence in adults and seniors from southeast São Paulo State, Brazil. An epidemiological survey was carried out among 1,475 dentate adults, ages 35 to 44 and 65 to 74, respectively, according to WHO criteria. Root surface caries prevalence was $15.6 \%$ and $31.8 \%$, for the 35-44 and 65-74-year groups, respectively. The average rate for decayed and/or filled roots was 0.36 for the middle-aged adult group and higher in males $(p<0.01)$, as compared to 0.82 for seniors, with no difference by gender. The average rate of decayed roots was 0.18 and 0.53 for middle-aged adults and seniors, respectively; in both groups the levels were higher among men $(p<0.01)$. Further studies on oral health, mainly on root surface conditions, are essential in both middleaged adults and senior citizens.
\end{abstract}

Oral Health; Dental Caries; Root Caries; Adult; Aged

\section{Introdução}

Grande enfoque tem sido dado ao declínio na experiência de cáries coronárias em crianças, no entanto, a literatura internacional é controversa em relação aos índices de cárie na população adulta e idosa. Enquanto trabalhos relatam melhora nas condições de saúde bucal com diminuição na ocorrência de cáries e edentulismo 1,2 , outros relatam populações geriátricas apresentando precária saúde bucal, com alta prevalência de cáries, inclusive radiculares 3,4 .

O desenvolvimento da cárie em superfície radicular é semelhante ao da lesão coronária, tendo como fatores etiológicos: exposição da superfície radicular ao ambiente bucal (hospedeiro), controle mecânico de placa deficiente (microbiota específica) e dieta cariogênica (substrato), que, interagindo em função do tempo, implicam formação e progressão de cárie 5,6.

Com relação às cáries radiculares, tem sido relatada uma ocorrência maior em adultos e idosos 3,4,7. Em algumas localidades, como sul da Austrália e Suécia, a ocorrência deste tipo de lesão vem sendo considerada como um fator de risco para perdas dentais na faixa etária acima de sessenta anos 8,9.

No Brasil, trabalhos descrevem elevado número de dentes extraídos 10,11, mas poucos estudos relatam a condição radicular da população. Em Piracicaba, Região Sudeste brasileira, encontrou-se, em 2002, prevalência de cárie 
radicular de $3,2 \%$, em indivíduos institucionalizados de 50 a 75 anos 4 .

Assim, torna-se cada vez mais relevante a verificação da condição radicular, visando ao direcionamento dos serviços, uma vez que as formas de prevenção e controle da doença são conhecidas e podem ser implantadas em serviços de saúde pública.

O objetivo deste estudo foi verificar a prevalência de cárie radicular em moradores de cidades representativas da região sudeste do Estado de São Paulo, na população adulta e idosa.

\section{Material e método}

Este estudo, transversal descritivo, utilizou dados secundários provenientes do Levantamento das Condições de Saúde Bucal para o Estado de São Paulo - 1998 12, abrangendo as 24 Direções Regionais de Saúde (DIR) do Estado, totalizando 133 municípios. A presente pesquisa trabalhou com resultados referentes às DIR das regiões de Campinas (DIR XII), Piracicaba (DIR XV), São João da Boa Vista (DIR XX) e Sorocaba (DIR XXIII).

Os códigos utilizados para a verificação de cárie radicular seguiram as recomendações da Organização Mundial da Saúde (OMS) 13, adaptados pela Secretaria Estadual de Saúde do Estado de São Paulo (SES-SP) 14. Para a verificação da prevalência de cárie radicular, foi utilizado o percentual de pessoas que apresentaram este tipo de lesão. Também se avaliou esta condição por meio das médias de raízes cariadas ou obturadas (CO-R), além das médias de cada um destes componentes em separado, média de raízes cariadas ( $C-R$ ), média de raízes obturadas $(\mathrm{O}-\mathrm{R})$ e média de raízes que, apesar de apresentarem recessão gengival, estavam hígidas no momento do exame (H-R). Estas variáveis foram analisadas segundo grupo etário (35-44 anos e 65 a 74 anos) e gênero (masculino e feminino).

O sorteio dos municípios participantes foi realizado de acordo com recomendações descritas no manual de instruções idealizado para o levantamento estadual 14. Assim, nas quatro DIR analisadas, foram sorteados 29 municípios: Jundiaí, Rio Claro, Mogi-Guaçu, Itapetininga, Valinhos, Leme, Divinolândia, Iperó, Pedra Bela, Vargem, Águas de São Pedro, Mombuca, Águas da Prata, Santo Antônio do Jardim, Itaóca, Barra do Chapéu, Hortolândia, Campo Limpo Paulista, Rio das Pedras, Caconde, Porto Feliz, Monte Alegre do Sul, Tuiuti, Corumbataí, Ipeúna, Estiva Gerbi, Holambra, Bom Sucesso de Itararé e Itapirapuã Paulista.
No estudo original, o tamanho amostral por idade e por município foi calculado segundo recomendação da OMS 15.

A calibração dos examinadores totalizou quarenta horas divididas entre discussões teóricas e atividades práticas simulando as situações que os examinadores encontrariam durante o trabalho prático. Em relação à técnica adotada, optou-se pela técnica do consenso, conforme recomendação da coordenação geral da pesquisa. Para a concordância interexaminadores, obtiveram-se os seguintes resultados: $99,2 \%$ para a DIR XII, $96,7 \%$ para a DIR XV, $84,0 \%$ para a DIR XX e $96,5 \%$ para a DIR XXIII 16. Valores acima de $90,0 \%$ são considerados aceitáveis, validando desta forma os resultados 16, no caso da DIR XX, que apresentou valor inferior a $90,0 \%$, a porcentagem de concordância encontrada é entendida como boa. Ressalta-se que a amplitude de variação para a porcentagem de concordância para o Estado de São Paulo, para todas as 24 DIR foi de 99,2\% - DIR XII, região de Campinas - a $82,5 \%$ - para a DIR II, Santo André.

A porcentagem de concordância intra-examinadores foi realizada durante a coleta dos dados, reexaminando-se $10,0 \%$ da amostra. Os valores encontrados foram de: $99,9 \%$ para a DIR XII, 95,0\% para a DIR XV, 99,8\% para as DIR XX, e na DIR XXIII não houve obtenção destes dados.

O número total de examinadores na região sudeste foi de 66 cirurgiões dentistas, divididos nas quatro DIR, sendo: 28 para a DIR XII (região de Campinas), 11 para a DIR XV (região de Piracicaba), 21 para a DIR XX (região de São João da Boa Vista) e 6 para a DIR XXIII (região de Sorocaba).

Os exames foram realizados em local com luz natural, utilizando-se espelho bucal plano e sonda CPI para levantamentos epidemiológicos 12 .

Os elementos amostrais foram constituídos de professores de primeiro grau e funcionários das escolas públicas e particulares, no grupo etário de 35-44 anos, examinados no próprio ambiente de trabalho, e de idosos, de 65-74 anos, usuários de serviços de saúde ou associados de grêmios, examinados nestas localidades. Para o presente trabalho, utilizaram-se dados apenas de indivíduos dentados.

Para o grupo de adultos, os indivíduos foram selecionados por intermédio da técnica de amostragem probabilística sistemática (nos municípios com menos de vinte escolas, todas foram incluídas para a seleção), em seguida, obteve-se a autorização para a realização dos exames, pelo termo de consentimento livre e esclarecido. Para os idosos, foram selecionados 
todos que freqüentaram grêmios ou serviços municipais de saúde nos meses de outubro a dezembro de 1998.

Os edêntulos e as fichas de exames epidemiológicos incompletas foram critérios de exclusão neste trabalho.

O total de exames válidos foi de 1.475 . Na análise estatística, utilizou-se o teste MannWhitney com nível de significância de $5 \%$, tendo em vista que a distribuição dos dados não era normal $(\mathrm{p}<0,05)$, verificada por um teste de normalidade.

Em se tratando de uma pesquisa que envolve seres humanos, esta se iniciou após a aprovação do Comitê de Ética em Pesquisa da Faculdade de Odontologia de Piracicaba (COEP 017/2003).

\section{Resultados}

O número de pessoas dentadas examinadas na região sudeste de São Paulo, segundo o grupo etário e gênero, é descrito na Tabela 1.

A média de dentes presentes na região sudeste, para os adultos foi de 21,3 , e neste grupo etário, analisando-se comparativamente os gêneros, encontrou-se 21,3, para ambos. Para os idosos esta média foi de 9,6; 10,6 para o gênero masculino e 8,8 para o feminino.

A prevalência de cárie radicular no sudeste de São Paulo representou 15,6\% (IC95\%: 10,0$21,2)$ da população examinada no grupo etário de 35 a 44 anos $(n=164)$ e $31,8 \%$ (IC95\%: 23,9$39,7)$ no grupo etário de 65 a 74 anos $(n=135)$, ou seja, esta prevalência foi obtida somente para a população dentada e, portanto, examinada para a condição cárie radicular.

A ocorrência de cárie radicular, segundo o grupo etário e gênero, pode ser visualizada na Tabela 2.

A porcentagem de pessoas segundo o grau de ataque de cárie, verificada pela média CO$\mathrm{R}$, de acordo com o grupo etário, é mostrada na Figura 1.

\section{Discussão}

Tratando-se de um estudo transversal que trabalhou com dados secundários provenientes do Levantamento das Condições de Saúde Bucal do Estado de São Paulo - 1998 14, ressalta-se que o tamanho amostral foi calculado segundo a prevalência de cárie coronária, sendo assim este se configura um estudo exploratório para cárie radicular. No grupo etário de idosos, houve uma média de dentes remanescentes por pessoa relativamente pequena, sugerindo-se

Tabela 1

Freqüência de pessoas dentadas segundo o grupo etário e sexo.

Região Sudeste do Estado de São Paulo, Brasil, 1998

\begin{tabular}{lccc}
\hline $\begin{array}{l}\text { Grupo etário } \\
\text { (anos) }\end{array}$ & $\begin{array}{c}\text { Masculino } \\
\mathbf{n}(\%)\end{array}$ & $\begin{array}{c}\text { Feminino } \\
\mathbf{n}(\%)\end{array}$ & $\begin{array}{c}\text { Total } \\
\mathbf{n}(\%)\end{array}$ \\
\hline 35 a 44 & $242(23,0)$ & $808(77,0)$ & $1.050(100,0)$ \\
65 a 74 & $186(43,8)$ & $239(56,2)$ & $425(100,0)$ \\
\hline
\end{tabular}

Tabela 2

Condição radicular (médias CO-R, C-R, O-R e H-R e intervalos de confiança) segundo o grupo etário e gênero.

Região Sudeste do Estado de São Paulo, Brasil, 1998.

\begin{tabular}{|c|c|c|c|c|c|c|c|c|}
\hline \multirow[t]{2}{*}{ Índices } & \multicolumn{4}{|c|}{35 a 44 anos } & \multicolumn{4}{|c|}{65 a 74 anos } \\
\hline & Geral & Masculino & Feminino & $p$ & Geral & Masculino & Feminino & $p$ \\
\hline CO-R & 0,36 & $0,49 a$ & $0,32 b$ & 0,005 & 0,82 & $1,01 \mathrm{a}$ & $0,67 a$ & 0,104 \\
\hline IC95\% & $0,28-0,44$ & $0,33-0,65$ & $0,23-0,41$ & & $0,64-1,00$ & $0,68-1,34$ & $0,48-0,86$ & \\
\hline C-R & 0,18 & $0,29 a$ & $0,15 b$ & $<0,001$ & 0,53 & $0,83^{a}$ & $0,29 b$ & $<0,001$ \\
\hline IC95\% & $0,12-0,24$ & $0,19-0,39$ & $0,08-0,22$ & & $0,38-0,68$ & $0,53-1,14$ & $0,16-0,41$ & \\
\hline O-R & 0,18 & $0,19 a$ & $0,17 a$ & 0,356 & 0,29 & $0,18^{a}$ & $0,38 b$ & 0,02 \\
\hline IC95\% & $0,13-0,23$ & $0,07-0,31$ & $0,12-0,22$ & & $0,20-0,38$ & $0,08-0,28$ & $0,24-0,52$ & \\
\hline $\mathrm{H}-\mathrm{R}$ & 3,65 & $5,52 a$ & $3,09 b$ & $<0,001$ & 4,50 & $5,24 a$ & $3,92 \mathrm{a}$ & 0,207 \\
\hline IC95\% & $3,43-3,87$ & $4,70-6,34$ & $2,74-3,43$ & & $3,94-5,06$ & $4,29-6,19$ & $3,26-4,58$ & \\
\hline
\end{tabular}

Nota: Números seguidos de letras diferentes distinguem entre si para cada variável,

segundo o gênero no nível de significância de $5 \%$ pelo teste de Mann-Whitney.

$\mathrm{CO}-\mathrm{R}=$ média de raízes cariadas e/ou restauradas; $\mathrm{C}-\mathrm{R}=$ média de raízes cariadas;

$\mathrm{O}-\mathrm{R}=$ média de raízes restauradas; $\mathrm{H}-\mathrm{R}=$ média de raízes hígidas 


\section{Figura 1}

Experiência de cárie radicular (CO-R), segundo o grupo etário. Região Sudeste do Estado de São Paulo, Brasil, 1998.

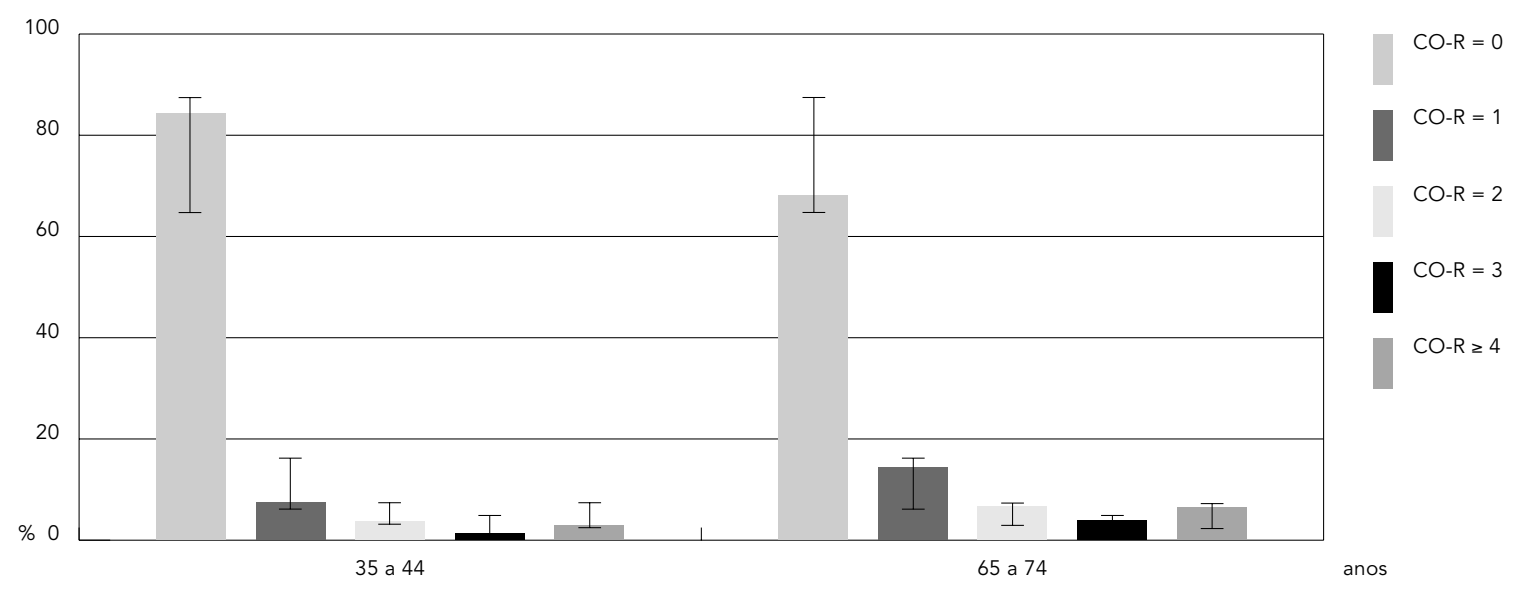

cautela na interpretação dos resultados para este grupo.

Outra limitação foi referente ao grupo de adultos, que consistiu de uma classe específica de trabalhadores (professores e funcionários de escolas públicas e particulares), e estes resultados não podem ser extrapolados para os adultos da região sudeste como um todo.

A prevalência de cárie radicular encontrada na população do sudeste apresentou-se relativamente baixa, embora o número médio de dentes por pessoa nesta população, especialmente entre os idosos, não tenha sido alta $(9,6$ dentes no grupo de 65 a 74 anos). Comparativamente, em relação a estudos internacionais, a prevalência de cárie radicular no sudeste de São Paulo pode ser considerada baixa, em relação a países como a França 3 e a Finlândia 17, onde as prevalências de cárie radicular encontradas foram de $88,0 \%$ e $52,0 \%$. Nestes países foram verificadas médias de dentes presentes bem maiores, sendo de 16,6 na França, 13,9 na Finlândia.

Analisando-se o CO-R, houve diferença entre os gêneros, na região sudeste, entre os adultos. Os homens apresentaram mais raízes cariadas $(C-R)$ e mais raízes expostas, porém hígidas $(\mathrm{H}-\mathrm{R})$, demonstrando neste último caso que, provavelmente, apresentam mais superfícies com recessão gengival. Entre os idosos, os homens apresentaram somente o componente cariado (C-R) maior que as mulheres (Tabela 2), o que também foi verificado em Helsink, em idosos 17 e em outros trabalhos 7 , fato que não significa que os homens têm uma pior saúde bucal, uma vez que a atividade de cárie radicular neste trabalho não foi verificada. Desta forma não se pode estar mensurando a proporção de raízes com cárie inativa. Além disto, tais resultados talvez indiquem que mulheres, no caso dos idosos, que apresentaram o componente obturado do CO-R maior, podem estar recebendo sobretratamento.

Em relação à exposição radicular, quando ocorre recessão da margem gengival, a superfície radicular fica exposta e, do ponto de vista da limpeza mecânica, esta região se apresenta como uma área de estagnação de placa 6 e, conseqüentemente há maior probabilidade de desenvolvimento de cárie radicular. Na região sudeste (Tabela 2), a média desta variável (H-R) foi alta em relação à experiência de cárie radicular em ambos os grupos etários, merecendo assim, cuidados por parte dos profissionais por intermédio da implementação de medidas de promoção de saúde e dos indivíduos no controle de placa.

A maioria das pessoas não apresentou experiência de cárie radicular (Figura 1). Entre os indivíduos em que a doença foi verificada, a maioria dos casos foi de somente uma lesão de cárie radicular em ambos os grupos etários, e uma pequena porcentagem $(3,0 \%$ e $6,6 \%$, para os adultos e idosos, respectivamente) apresentou quatro ou mais raízes cariadas.

Levantamentos epidemiológicos que estudam as condições radiculares apresentam uma certa vulnerabilidade ou limitação nos resulta- 
dos. A estimativa de prevalência de cárie em superfície radicular pode ser dificultada pela presença de restaurações nesta superfície, visto que elas podem ter sido realizadas por motivos de origem não cariosa 18. Apesar disso, quando apenas lesões de cárie não tratadas são diagnosticadas, a confiabilidade do exame é reduzida consideravelmente, e em estudos de incidência, podem estar, segundo Clarkson 19, subestimando mais de dois terços de sua incidência 5,19 .

Apesar desta vulnerabilidade dos levantamentos epidemiológicos nestas idades, alguns aspectos como: média de raízes hígidas e média de dentes presentes foram ressaltadas e a sua verificação em estudos que avaliem a condição radicular devem ser verificadas.

\section{Resumo}

Observa-se uma ocorrência maior de cárie radicular nas últimas décadas, sendo este tipo de lesão mais comum em adultos e idosos. Em virtude disso, o objetivo deste estudo foi avaliar a prevalência de cáries radiculares em adultos e idosos da região Sudeste do Estado de São Paulo, Brasil. Exames epidemiológicos foram realizados seguindo recomendações da OMS, sendo examinadas 1.475 pessoas dentadas, dos grupos etários de 35 a 44 e de 65 a 74 anos. A prevalência de cáries radiculares foi de 15,6\% e 31,8\%, respectivamente, para 35 a 44 e 65 a 74 anos. A média de raízes cariadas elou restauradas foi de 0,36 para os adultos, maior entre os homens $(p<0,01)$ e de 0,82 para os idosos, sem diferença no gênero. A média de raízes cariadas foi de 0,18 e 0,53, respectivamente, para adultos e idosos; em ambos os grupos os valores foram maiores entre os homens ( $p<0,01)$. Em razão das condições observadas, ressalta-se a necessidade de mais estudos relacionados com a saúde bucal de adultos e idosos, sobretudo da condição radicular.

Saúde Bucal; Cárie Dentária; Cárie Radicular; Adultos; Idoso
Desse modo, o declínio na prevalência de edentulismo e o aumento no número de pessoas que mantêm seus dentes naturais por período maior de tempo provavelmente podem resultar em um aumento do risco de desenvolvimento de cárie radicular no futuro, implicando necessidade de capacitação dos profissionais não somente para o diagnóstico correto da cárie radicular, mas também para alternativas e indicações de tratamento, pois se espera um contingente cada vez maior de pessoas com presença de cáries radiculares. Assim, há a necessidade de se enfatizar estudos relacionados com a saúde bucal de adultos e idosos, sobretudo da condição radicular.

\section{Colaboradores}

L. B. Rihs organizou o banco de dados. M. L. R. Sousa contribuiu na calibração da equipe de examinadores. R. S. Wada realizou a análise estatística. Todos os autores realizaram em conjunto todas as demais etapas do trabalho, desde a análise dos resultados até a redação do artigo científico.

\section{Agradecimentos}

À Maria Candelária Soares, Coordenadora Estadual de Saúde Bucal, por disponibilizar os dados. Aos Coordenadores de Saúde Bucal das DIR envolvidas neste estudo, Nádja M. Abdala, Márcia Regina Angeli Jordão, Airton Dias Pascoal e Wilson Gonçalves, que autorizaram a utilização dos dados dos municípios envolvidos. Aos voluntários, que aceitaram participar do levantamento. 


\section{Referências}

1. Fure S, Zickert I. Incidence of tooth loss and dental caries in 60-, 70- and 80-year-old Swedish individuals. Community Dent Oral Epidemiol 1997; 25:137-42.

2. Kalsbeek H, Truin GJ, van Rossum GM, van Rijkom HM, Poorterman JH, Verrips GH. Trends in caries prevalence in Dutch adults between 1983 and 1995. Caries Res 1998; 32:160-5.

3. Guivante-Nabet C, Tavernier M, Trevoux C, Berdal A. Active and inactive caries lesions in a selected elderly institutionalized French population. Int Dent J 1998; 48:111-22.

4. Meneghim MC, Pereira AC, Silva, FRB. Prevalência de cárie radicular e condição periodontal em uma população dosa institucionalizada de Piracicaba - SP. Pesqui Odontol Bras 2002; 16:50-6.

5. Banting DW. The diagnosis of root caries. J Dent Educ 2001; 65:991-6.

6. Thylstrup A, Fejerskov O. Cariologia clínica. São Paulo: Editora Santos; 2001.

7. Vehkalahti MM, Virbic VL, Peric LM, Matvoz ES. Oral hygiene and root caries occurrence in Slovenian adults. Int Dent J 1997; 47:26-31.

8. Slade GD, Spencer AJ. Distribution of coronal and root caries experience among persons aged 60+ in South Australia. Aust Dent J 1997; 42:178-84.

9. Fure S. Five-year incidence of caries, salivary and microbial conditions in 60-, 70- and 80-year-old Swedish individuals. Caries Res 1998; 32:166-74.

10. Ministério da Saúde. Levantamento epidemiológico em saúde bucal: Brasil, zona urbana, 1986. Brasília: Divisão Nacional de Saúde Bucal, Ministério da Saúde; 1988.
11. Fernandes RAC, Silva SRC, Watanabe MGC, Pereira AC, Martildes MLR. Uso e necessidade de prótese dentária em idosos que demandam um centro de saúde. Rev Bras Odontol 1997; 54:107-10.

12. Secretaria de Estado da Saúde de São Paulo. Levantamento epidemiológico em saúde bucal: Estado de São Paulo, 1998. São Paulo: Secretaria de Estado da Saúde de São Paulo; 1999.

13. World Health Organization. Oral health surveys: basic methods. Geneva: World Health Organization; 1997.

14. Secretaria de Estado da Saúde de São Paulo. Levantamento das condições de saúde bucal - Estado de São Paulo, 1998: caderno de instruções. São Paulo: Secretaria de Estado da Saúde de São Paulo; 1998.

15. World Health Organization. Basic methods. Geneva: World Health Organization; 1987.

16. Frias AC. Estudo de confiabilidade do levantamento epidemiológico de saúde bucal - Estado de São Paulo, 1998 [Dissertação de Mestrado]. São Paulo: Faculdade de Saúde Pública, Universidade de São Paulo. 2000.

17. Narhi TO, Vehkalahti MM, Siukosaari P, Ainamo A. Salivary findings, daily medication and root caries in the old elderly. Caries Res 1998; 32:5-9.

18. Walls AWG, Silver PT, Steele JG. Impact of treatment provision on the epidemiological recording of root caries. Eur J Oral Sci 2000; 108:3-8.

19. Clarkson JE. Epidemiology of root caries. Am J Dent 1995; 8:329-34.

Recebido em 14/Jan/2004

Versão final reapresentada em 13/Set/2004

Aprovado em 14/Set/2004 